\title{
Single Tripyridyl-Triazine Molecular Junction with Multiple Binding Sites
}

\author{
Madoka Iwane, Shintaro Fujii,* Tomoaki Nishino, Manabu Kiguchi* \\ Department of Chemistry, Tokyo Institute of Technology, \\ 2-12-1 Ookayama, Meguro-ku, Tokyo 152-8551, Japan
}

Table of contents

1. Individual conductance traces

2. Additional data for blank measurements

3. Gap distance analysis

4. DFT calculations

5. STM-BJ experiments for molecular junctions of $4,4^{\prime}$ '-bipyridine 


\section{Individual conductance traces}

Figure S1 shows conductance traces of blank and the TPTZ-molecular junction using the two different linear current amplifiers $(1 \mu \mathrm{A} / \mathrm{V}$ and $10 \mathrm{nA} / \mathrm{V})$ in separated experiments. The upper conductance limits were $1.3 G_{0}$ and $0.027 G_{0}$ for the $1 \mu \mathrm{A} / \mathrm{V}$ and $10 \mathrm{nA} / \mathrm{V}$-current amplifiers. In the presence of the molecule plateau-regions in the conductance traces are elongated, which is mostly due to formation of the molecular junctions as well as the interaction between the molecules and the Au contact.
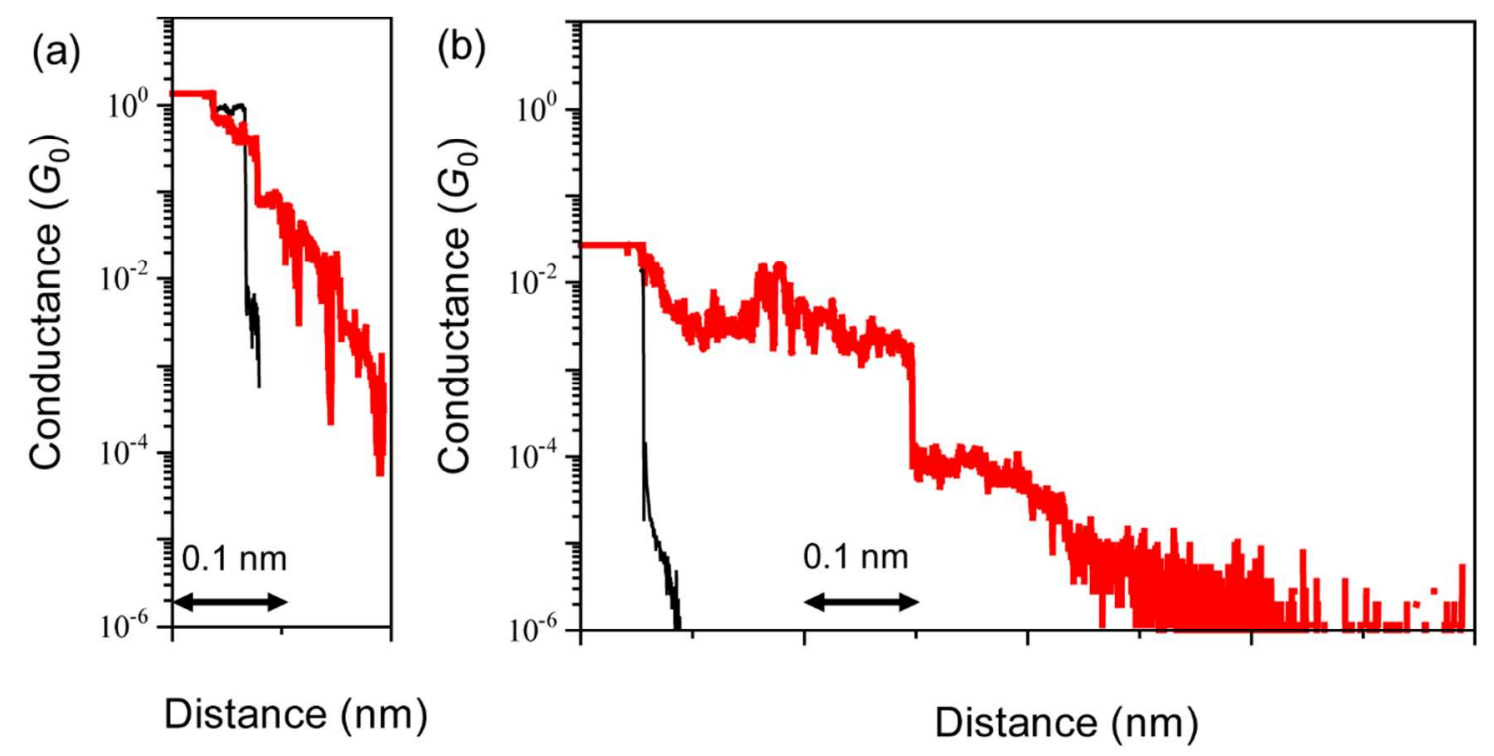

Figure S1. Conductance traces for blank (back) and the TPTZ-molecular junction (red) using the current amplifiers with (a) $1 \mu \mathrm{A} / \mathrm{V}$ and (b) $10 \mathrm{nA} / \mathrm{V}$ at the bias voltage of $100 \mathrm{mV}$ and $50 \mathrm{mV}$, respectively. Scale bars are $0.1 \mathrm{~nm}$. 


\section{Additional data for blank measurements}

\subsection{D conductance histograms}

Figure S2 shows 2D conductance histograms for $\mathrm{Au}$ atomic contacts in the absence of the TPTZ molecule (blank). Each histogram is built from 1,000 conductance traces. For the construction of the 2D histogram, the first identified data point at a threshold conductance value was set to "distance $=0$ " in each conductance trace to overlap the conductance traces in the $2 \mathrm{D}$ histograms. For the higher (lower) conductance regime [with the $1 \mu \mathrm{A} / \mathrm{V}(10 \mathrm{nA} / \mathrm{V})$ current amplifier], the threshold conductance was set to $0.5 G_{0}\left(0.026 G_{0}\right)$. The blank-histograms are characterized by background tunneling conductance (current). See also 1D conductance histograms in Figure S3.
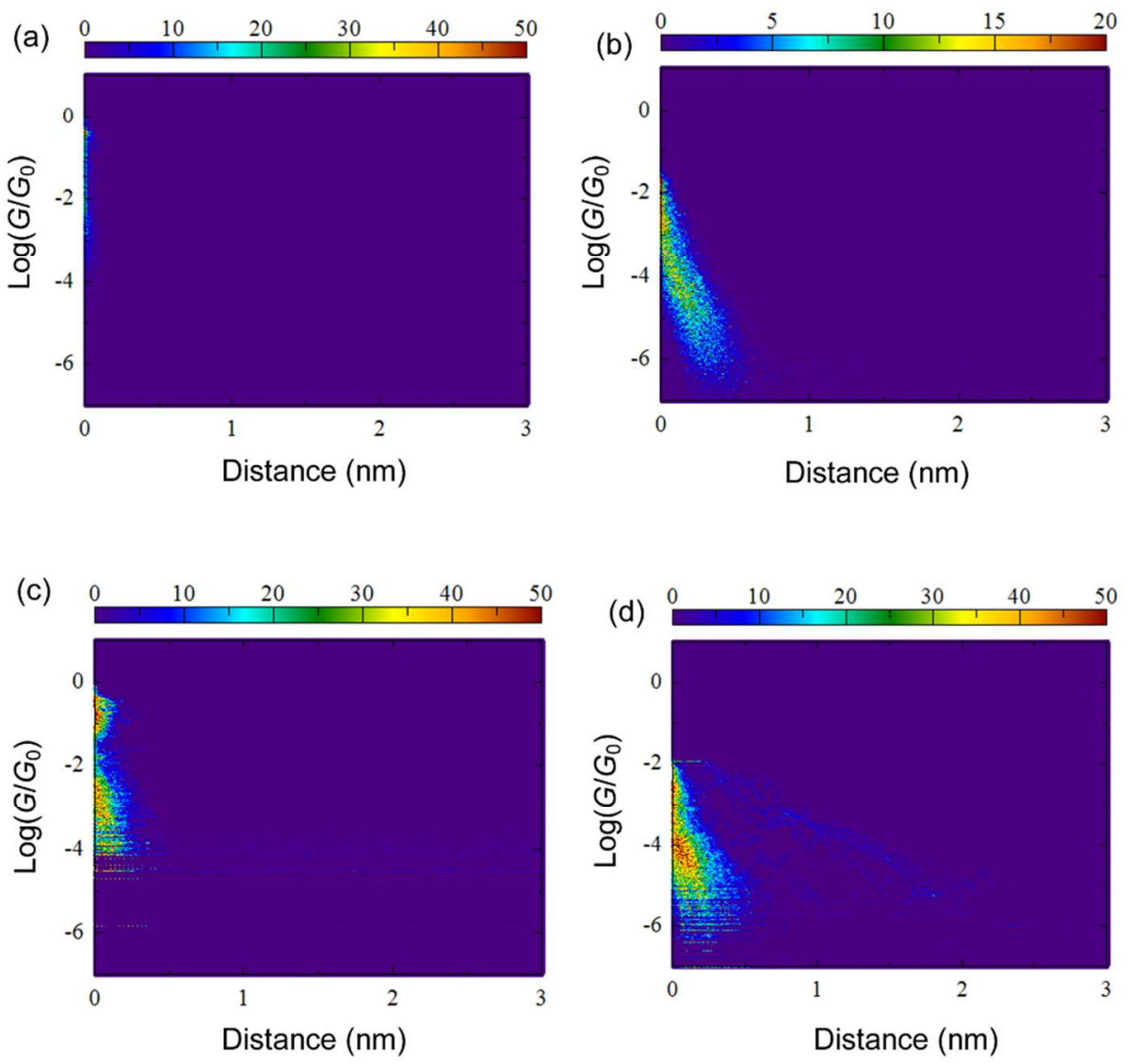

Figure S2. 2D conductance histograms for blank using the current amplifiers with (a)(c) $1 \mu \mathrm{A} / \mathrm{V}$ and (b)(d) $10 \mathrm{nA} / \mathrm{V}$ at the bias voltage of 50 100 mV. A linear bin-size of $0.01 \mathrm{~nm}$ and a logarithmic Y-binsize for $\Delta \log \left(G / G_{0}\right)$ of 0.01 were used for construction of conductance histograms. 


\subsection{D Conductance histogram}
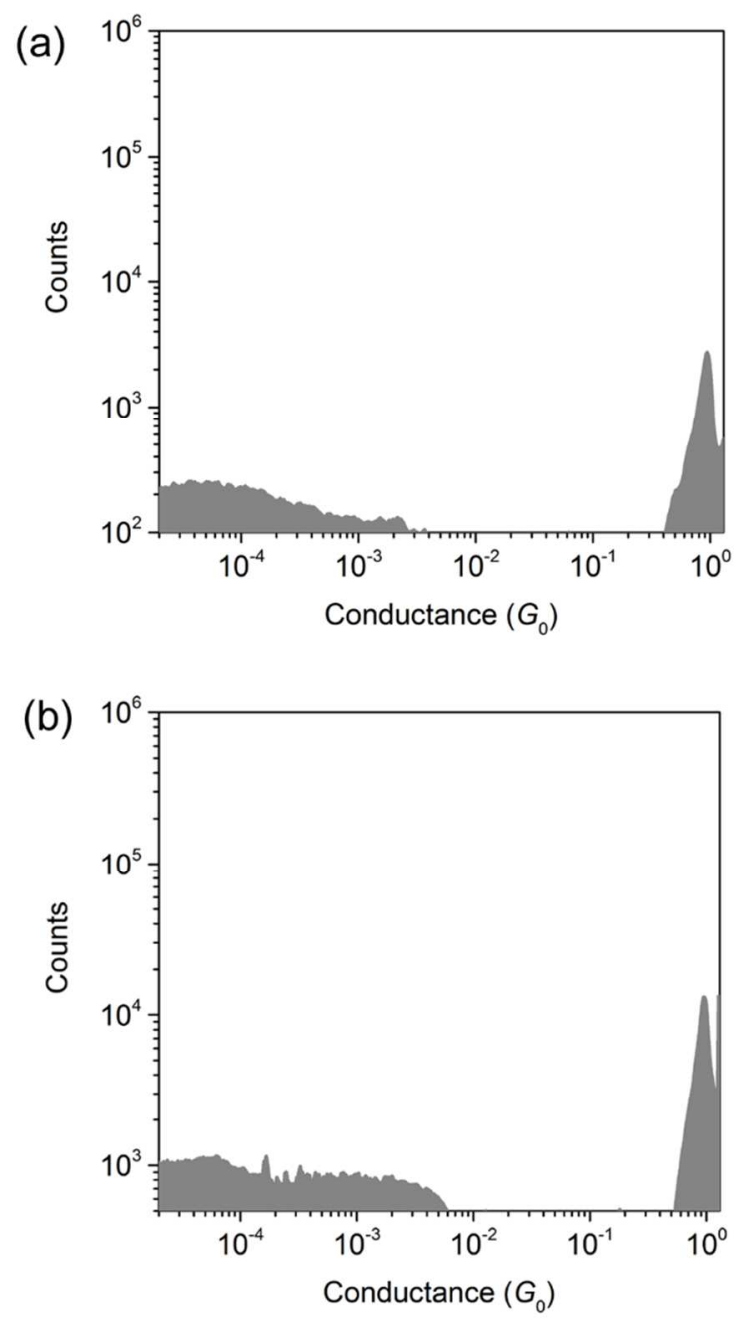

Figure S3. Conductance histograms for blank, which are constructed from 1,000 conductance traces. The histograms of (a) and (b), respectively, correspond the data set in Figures S2a,b and Figures S2c,d. A logarithmic bin-size for $\Delta \log \left(G / G_{0}\right)$ of 0.01 were used for conductance histograms. 


\subsection{Comparison between blank and the molecular conductance-histogram.}

Figures S4a-c show magnified 2D conductance histograms for blank and molecular junction. In the blank data in Figure S4a, there is no preferential conductance distribution below $1 G_{0}$, while we can notice conductance distribution above $0.1 G_{0}$ and a large tunneling background below $10^{-2} G_{0}$ for a blank data in Figure S4b. The conductance distribution above $0.1 G_{0}$ can be understood by the interaction and adsorption of small molecules such as $\mathrm{O}_{2}$ on the Au contact. ${ }^{\mathrm{S} 1, \mathrm{~S} 2}$ This effect of can appear in the conductance histogram of the TPTZ-molecular junction, in which additional conductance distribution around and below $0.1 G_{0}$ is noticeable in Figure S4c. Such molecular conductance can be recognized in the 1D conductance histogram in Figure S4d. By comparing the blank and the molecular conductance-data, we concluded that the additional conductance appears near and below $0.1 G_{0}$ is attributable to the formation of the TPTZ-molecular junctions.
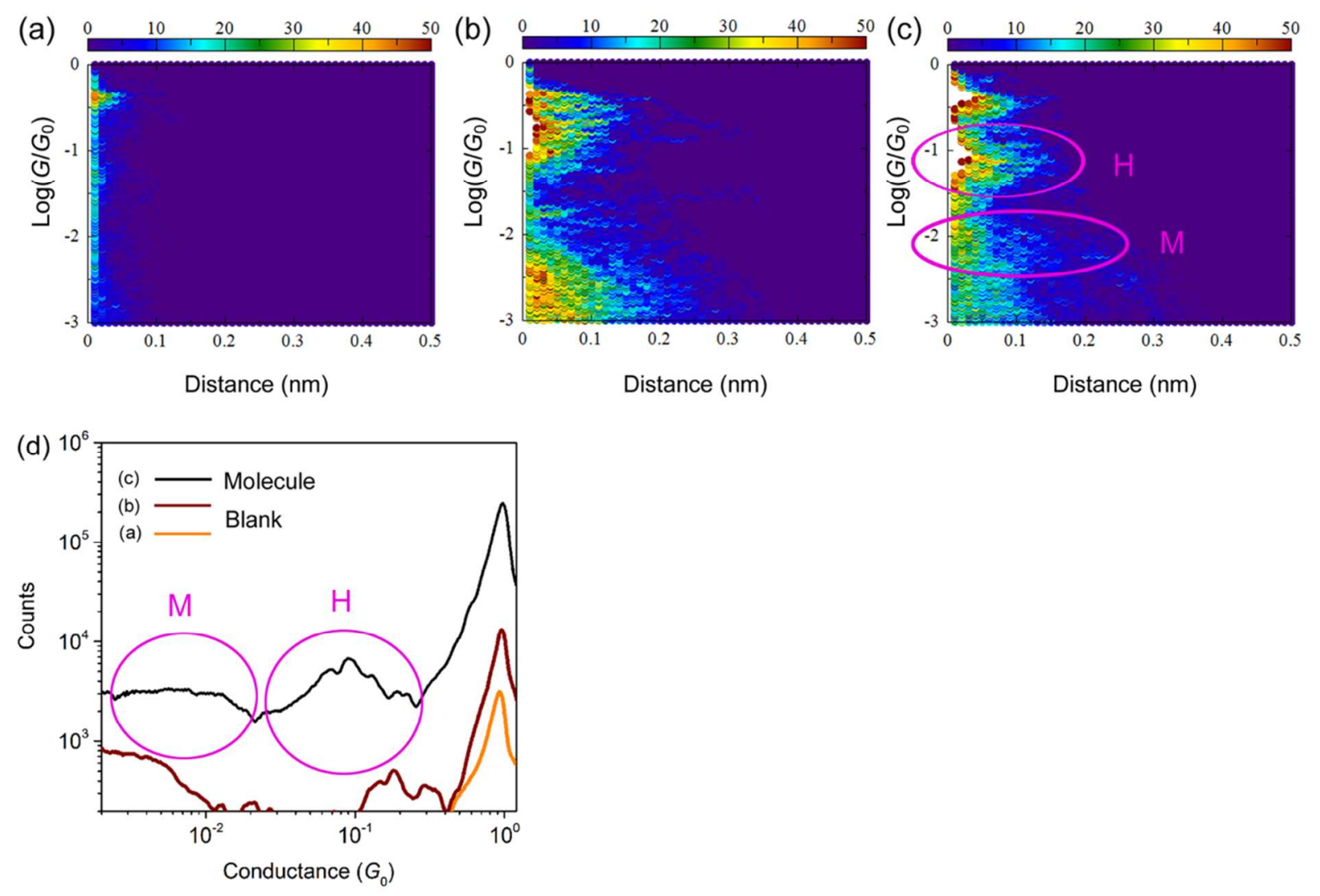

Figure S4. (a-c) 2D conductance histograms for $(a, b)$ blank and (c) the molecular junction using the current amplifiers with $1 \mu \mathrm{A} / \mathrm{V}$ at the bias voltage of $100 \mathrm{mV}$. The histograms in (a) and (b) are 
magnified blank-histograms of those in Figure 3a and Figure S2c. The histogram in (c) is magnified molecular-histogram of that in Figure 3c. (d) 1D conductance histograms for the data used in (a)-(c). A logarithmic bin-size for $\Delta \log \left(G / G_{0}\right)$ of 0.01 were used for conductance histograms. In (c) and (d), conductance distributions corresponding to the $\mathrm{H}$ and $\mathrm{M}$ states (see main text) are marked by circles. 


\section{Gap distance analysis}

Figure S5 shows histograms of the gap distance for the Au contacts and TPTZ-molecular junctions, which are constructed from 1,000 conductance traces recorded at higher and lower conductance regimes with the $1 \mu \mathrm{A} / \mathrm{V}$ and $10 \mathrm{nA} / \mathrm{V}$-current amplifiers. The gap distance was calculated as the distance between points (set points) at which the conductance drops below $0.8 G_{0}$ and $0.026 G_{0}$ in the higher conductance regime (Figure S5a). It should be noted here that the conductance of $0.8 G_{0}$ and $0.026 G_{0}$, respectively, corresponds to the breakage of an atomic Au contact and the lower conductance-limit of the $1 \mu \mathrm{A} / \mathrm{V}$-current amplifier. In a similar manner, the set points are $0.026 G_{0}$ and $0.1 \times 10^{-4} G_{0}$ in the lower conductance regime (Figure S5b). The longer gap distance was found for the molecular junctions, which corresponds to the formation of the TPTZ-molecular junctions. Average gap distances of the Au junctions are calculated to be $0.03 \mathrm{~nm}$ (Figure S5a) and $0.13 \mathrm{~nm}$ (Figure S5b). For the molecular junctions, average gap distances are $0.05 \mathrm{~nm}$ (Figure S5a) and $0.80 \mathrm{~nm}$ (Figure S5b) within the corresponding conductance windows.

In the molecular conductance measurement, the gap size of $0.05 \mathrm{~nm}$ within the conductance window from $0.026 G_{0}$ to $0.8 G_{0}$ is associated with the length of the molecular junction after the breakage of the $\mathrm{Au}$ contact. To evaluate actual length of the molecular junction, the snapback effect of the $\mathrm{Au}$ electrodes $^{\mathrm{S} 3, \mathrm{~S} 4}$ has to be taken into account. After the breakage of the Au contact, the resulting electrodes are subject to the elastic response, in which the electrodes are snapped back to enlarge the electrode-gap distance. For the Au contact, snapback distance is reported to be $c a .0 .4^{\mathrm{S} 3}$ and $0.5 \mathrm{~nm} .{ }^{\mathrm{S} 3}$

Figure 5 shows the distribution of the gap distance for H, M, and L states. The peak-gap distance is 0.01 , 0.30 and $0.67 \mathrm{~nm}$ for the $\mathrm{H}, \mathrm{M}$, and L states, respectively. The gap distance was calculated as the length of the molecular conductance-plateau within the conductance-window between $0.3 G_{0}$ and $0.026 G_{0}$ for H state. The windows are between $0.026 G_{0}$ and $0.8 \times 10^{-3} G_{0}$ for M state, $0.026 G_{0}$ and $1.0 \times 10^{-5} G_{0}$ for $\mathrm{L}$ states (see Figure 5 and corresponding text). By considering the snapback effect, the actual gap size of 
the molecular junction in the $\mathrm{H}$ state is estimated to be $0.01+0.5 \approx 0.5 \mathrm{~nm}$. To evaluate the actual gap size of the molecular junction in M and L states, offset-gap distance before the conductance drops to the upper limit of the $10 \mathrm{nA} / \mathrm{V}$-current amplifier (i.e., $0.026 G_{0}$ ) should be included. The estimated offsetgap distance is $0.05 \mathrm{~nm}$ (Figure S5a). By considering both of the snapback effect and the offset-gap distance, the actual gap size of the molecular junction in the $\mathrm{M}$ and $\mathrm{L}$ states are estimated to be $0.30+$ $0.5+0.05 \approx 0.9 \mathrm{~nm}$ and $0.67+0.5+0.05 \approx 1.2 \mathrm{~nm}$. Table S1 shows summary of the estimation of the actual gap distance.

Previous theoretical study on $\pi$-conjugated planar molecule (pentacene) adsorbed on Au(111) revealed that inter plane distance between Au surface and the $\pi$-conjugated planar molecule is $c a .0 .3 \mathrm{~nm}$, which is based on density functional theory with dispersion corrections. ${ }^{\mathrm{S} 5}$ On the basis of the inter plane distance of $0.3 \mathrm{~nm}$, “Au- $\pi$ ” bond-distance (i.e., inter plane distance between Au surface and the planar TPTZ molecule) in the TPTZ-molecular junction can be estimated to be $c a .0 .6 \mathrm{~nm}(=2 \times “ \mathrm{Au}-\pi$ " $=$ $2 \times 0.3 \mathrm{~nm}$ ). Previous X-ray crystallographic study on a metal complex of the TPTZ molecules reported that the inter-ring distances between triazine-pyridine and pyridine-pyridine are 0.41 and $0.72 \mathrm{~nm},{ }^{21} \mathrm{By}$ combining the estimated "Au- $\pi$ " bond-distance of $2 \times 0.3 \mathrm{~nm}$ and the inter-ring distances, the gap distance can be estimated to be $\mathrm{L}=0.60 \mathrm{~nm}, \mathrm{M}=0.6+0.41=1.01 \mathrm{~nm}$, and $\mathrm{L}=0.6+0.72=1.32 \mathrm{~nm}$. 

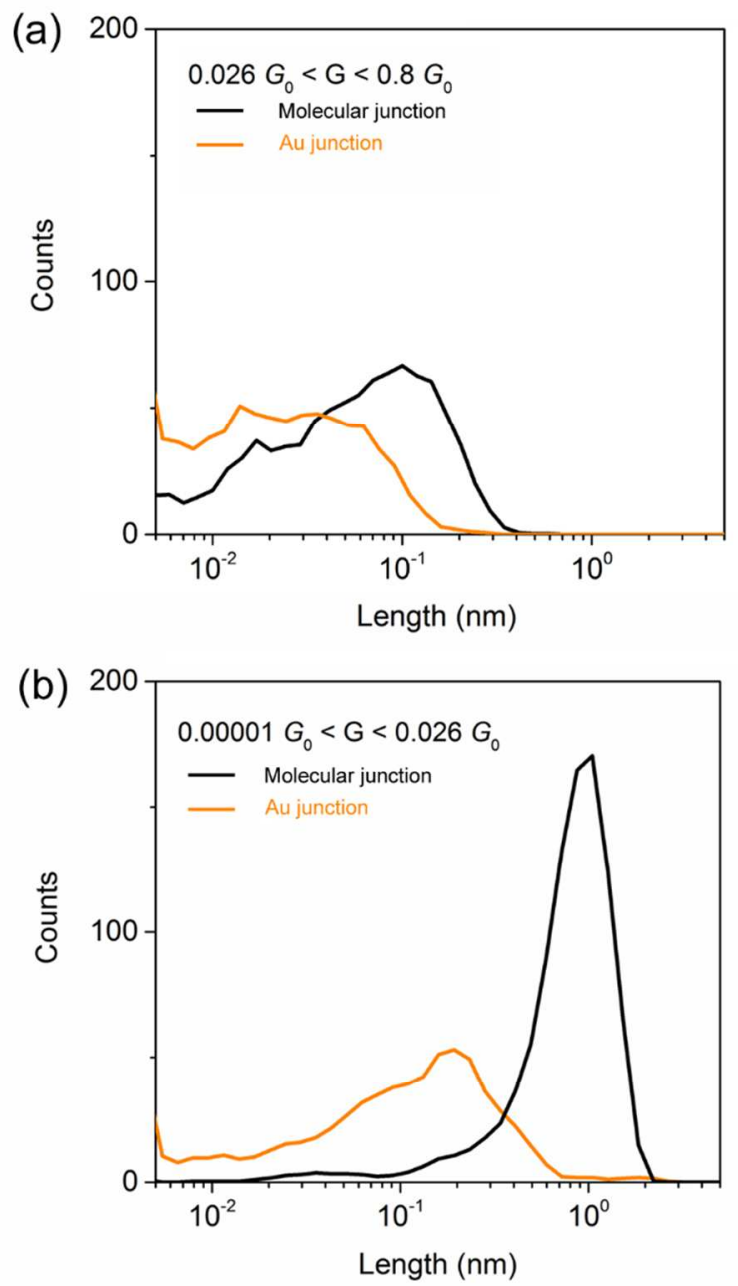

Figure S5. Histograms of the gap distance for the Au contacts (orange) and TPTZ molecular junctions (black), which are constructed from 1,000 conductance traces recorded with the (a) $1 \mu \mathrm{A} / \mathrm{V}$ and (b) 10 $\mathrm{nA} / \mathrm{V}$-current amplifiers. The same data sets of the conductance traces in Figure 3 are used here.

\begin{tabular}{|c|c|c|c|c|}
\hline & $\begin{array}{l}\text { Calculated gap size / nm } \\
\text { (Figure 5) }\end{array}$ & Snapback effect / $\mathbf{n m}$ & Offset-gap size / $\mathbf{n m}$ & Actual gap distance / $\mathbf{n m}$ \\
\hline $\mathbf{H}$ & 0.01 & 0.5 & & $\approx 0.5$ \\
\hline $\mathbf{M}$ & 0.30 & 0.5 & 0.05 & $\approx 0.9$ \\
\hline $\mathbf{L}$ & 0.67 & 0.5 & 0.05 & $\approx 1.2$ \\
\hline
\end{tabular}

Table S1. Summary of the estimation of the actual gap distance 


\section{DFT calculations}

Density functional theory (DFT) calculations were carried out using the Gaussian 09 program package. ${ }^{\mathrm{S} 6}$ Geometry optimizations were performed at the B3LYP/6-31G(d) level of theory. Figure S6 shows optimized structure of TPTZ. The ortho-N atom in the pyridyl group in TPTZ is spatially hidden from the surrounding vacuum. The calculated the inter-ring distances between triazine-pyridine and pyridine-pyridine are 0.42 and $0.73 \mathrm{~nm}$, which is in good agreement with those found in literature. ${ }^{21}$
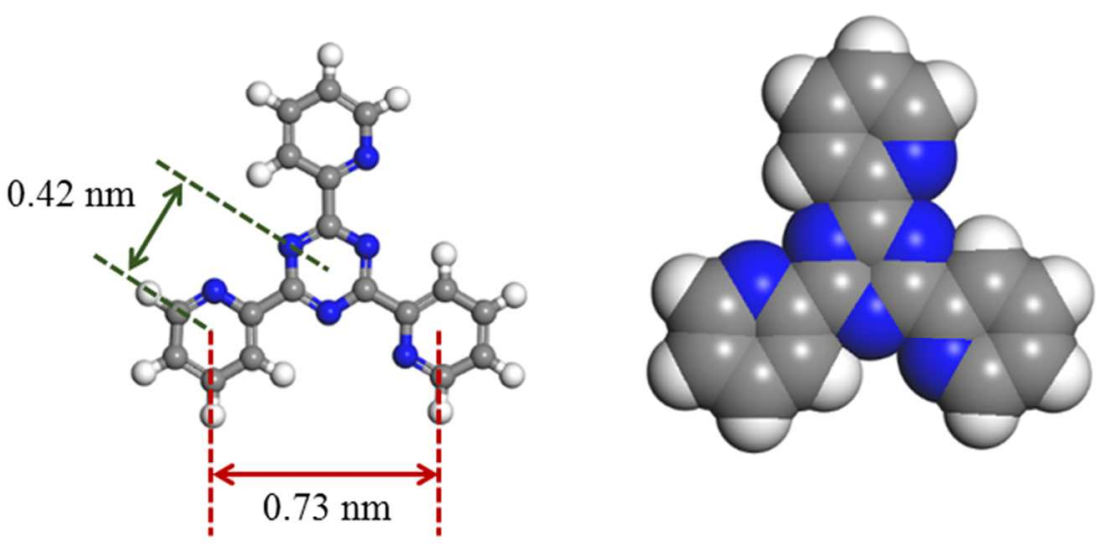

Figure S6. Optimized structure of TPTZ (right; ball and stick model and left; space filling model). White, grey and blue balls correspond to $\mathrm{H}, \mathrm{C}$ and $\mathrm{N}$ atoms. 


\section{STM-BJ experiments for molecular junctions of 4,4'-bipyridine}

To confirm the methodology of our STM-BJ technique, STM-BJ experiments were performed for the molecular junctions of 4,4'-bipyridine (BPY). BPY gives high probability of the molecular junctionformation and the conductance values have been confirmed in different laboratories (e.g., see refs S7S9). First, we performed STM-BJ experiment on a clean Au(111) substrate to collect several thousands of conductance traces. Then a drop of $6.4 \mathrm{mM}$ BPY ethanol solution was deposited on the $\mathrm{Au}(111)$, on which we measured conductance of the BPY molecular junctions. Figure S7 shows 1D conductance histograms for $\mathrm{Au}$ contacts with and without $\mathrm{BPY}$, which were constructed from 1,000 conductance traces. The histograms for the clean $\mathrm{Au}(111)$ reproduce the results in Fig. 4a. To see conductance distribution around $1 G_{0}$-region as well as the lower molecular conductance range below ca.0.1 $G_{0}$ (see Supporting information 2.3 for a detail in the molecular conductance range), the y-scale is changed from a linear scale (Fig. S7a) to a log scale (Fig. S7b). As is clearly visible in the conductance histograms in Fig. S7a, intensity of $1 G_{0}$-peaks of the Au-monoatomic contact was increased after the deposition of BPY, which can be explained by molecular adsorption on the Au atomic contacts. ${ }^{\text {S10,S11 }}$ Since the intensity of the $1 G_{0}$ peak can be affected by the molecular adsorption, normalization of the counts based on the $1 G_{0}$ peak-intensity in the histograms was not performed in this study. In the molecular conductance range, significant increase in the counts can be recognized after the molecular disposition. The increase in the intensity of the $1 G_{0}$-peak and the increase in the counts in the molecular conductance range after the molecular deposition (Fig. S7) nicely match up with the trends observed in the histograms in the main paper (Fig. 4).

Next, we analyze BPY-molecular conductance-peaks found in the conductance histograms. Figure S8a shows a magnified conductance histogram in Fig. S7b. Two molecular conductance-peaks are apparent around $10^{-3} G_{0}$ and $10^{-4} G_{0}$, which are in good agreement with the molecular conductances in literature. ${ }^{\mathrm{S} 9}$ It should be noted that the two molecular conductance were due to the BPY molecular junctions with different Au-BPY contact geometries. ${ }^{\mathrm{S} 9}$ In addition to the two molecular conductance- 
peaks, small or faint conductance distributions can be seen in the conductance range around $10^{-2} G_{0}$. Figure S8b shows a linear-bin conductance histogram constructed from the same data used in Fig. S7, in which small increase in counts around $0.01 G_{0}$ can be recognized. The conductance distribution can correspond to the molecular conductance of $0.01 G_{0}$ in literature ${ }^{\mathrm{S} 7}$ Our experiments reproduced the molecular conductance of BYP in literature, which assess the applicability of the present STM-BJ technique.

(a)

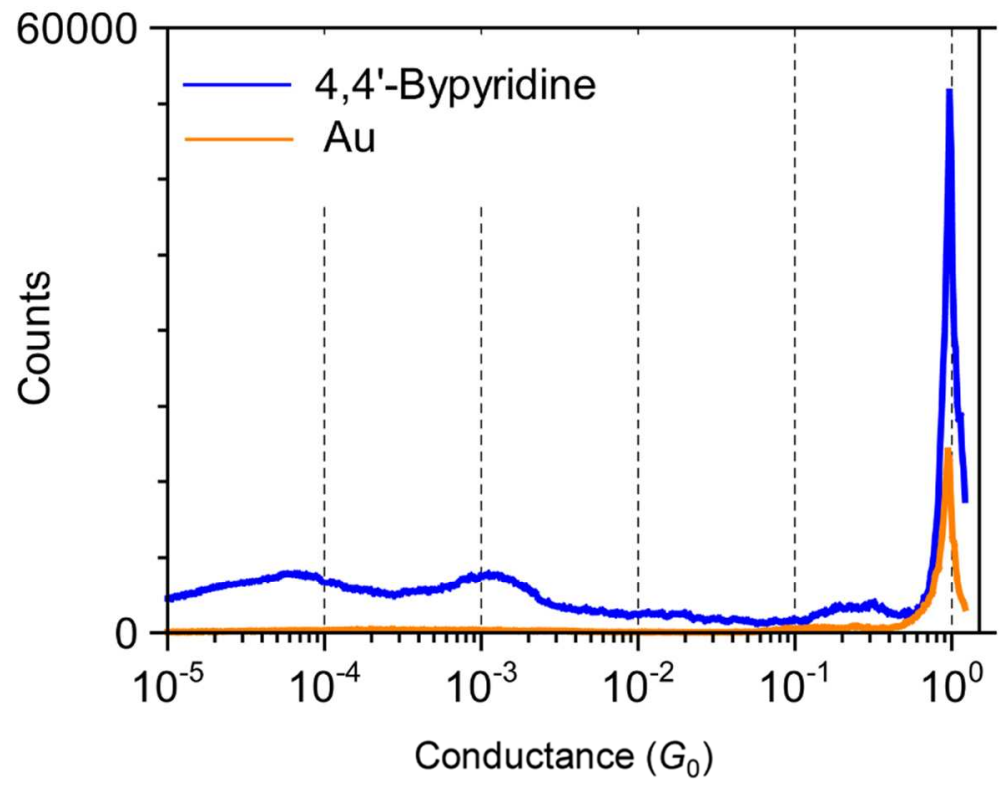

(b)

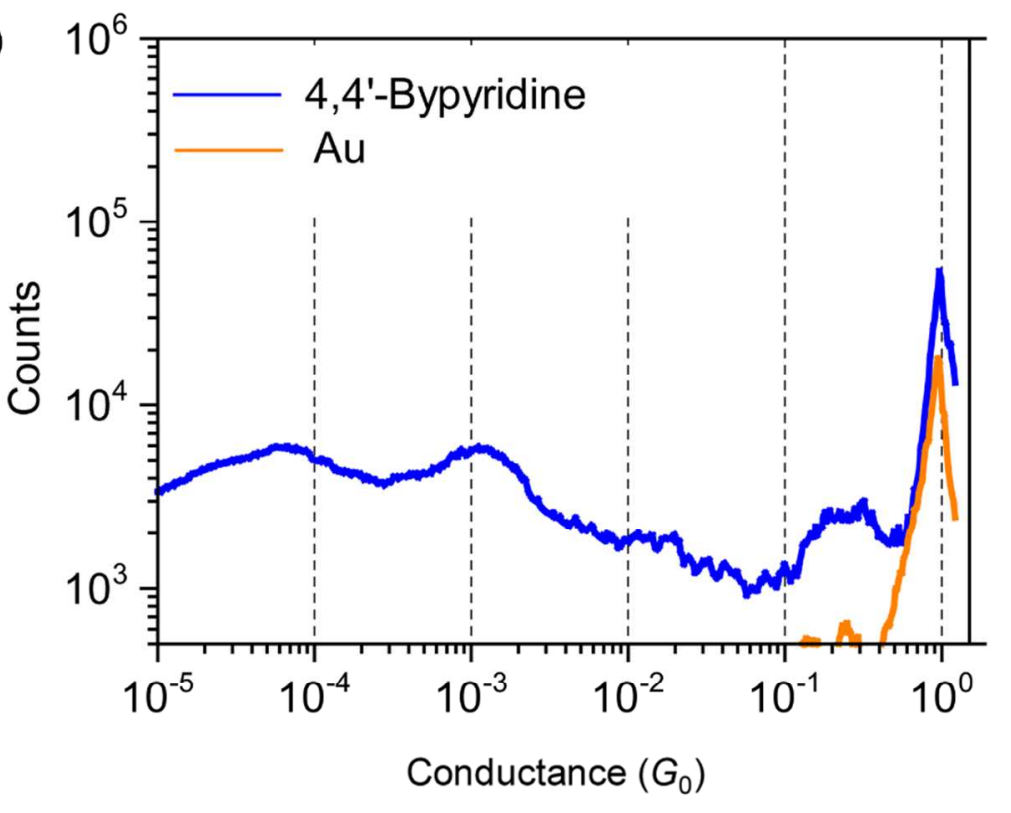


Figure S7. 1D conductance histograms for BPY molecular junctions and Au junctions without BPY $\left(V_{\mathrm{s}}\right.$ $=20 \sim 100 \mathrm{mV})$. A logarithmic bin-size for $\Delta \log \left(G / G_{0}\right)$ of 0.01 were used for conductance histograms. Linear and $\log \mathrm{Y}$-scales are used in (a) and (b), respectively.
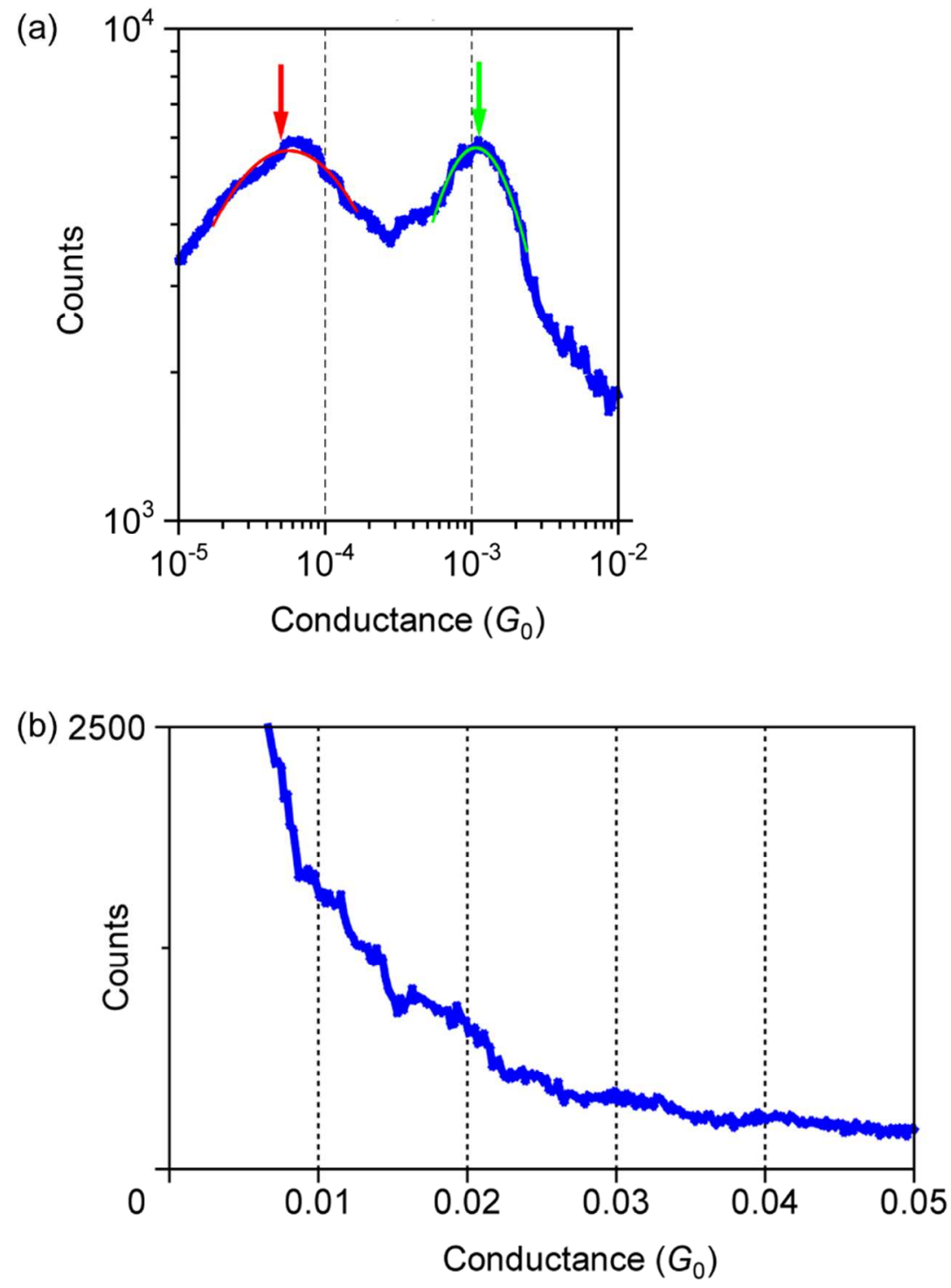

Figure S8. (a) Expanded conductance histogram in Fig. S7b in the conductance range from $10^{-5}$ to $10^{-2}$ $G_{0}$. The conductance peaks are denoted by arrows $\left(1.1 \times 10^{-3} G_{0}\right.$ and $\left.5.7 \times 10^{-5} G_{0}\right)$. The peak positions are obtained by Gaussian fitting. (b) Linear binned-1D conductance histogram in the conductance range from 0 to $0.05 G_{0}$. A linear bin-size of $0.002 G_{0}$ is used. 


\section{REFERENCES}

(S1) Thijssen, W. H. A.; Marjenburgh, D.; Bremmer, R. H.; van Ruitenbeek, J. M. Oxygen-Enhanced Atomic Chain Formation. Phys. Rev. Lett. 2006, 96, 026806.

(S2) Kiguchi, M.; Djukic, D.; van Ruitenbeek, J. M. The Effect of Bonding of a CO Molecule on the Conductance of Atomic Metal Wires. Nanotechnology 2007, 18, 035205.

(S3) Zhao, J.; Murakoshi, K.; Yin, X.; Kiguchi, M.; Guo, Y.; Wang, N.; Liang, S.; Liu, H. Dynamic Characterization of the Postbreaking Behavior of a Nanowire. J. Phys. Chem. C 2008, 112, 20088-20094.

(S4) Hong, W.; Manrique, D. Z.; Moreno-García, P.; Gulcur, M.; Mishchenko, A.; Lambert, C. J.; Bryce, M. R.; Wandlowski, T. Single Molecular Conductance of Tolanes: Experimental and Theoretical Study on the Junction Evolution Dependent on the Anchoring Group. J. Am. Chem. Soc. 2012, 134, 2292-2304.

(S5) Toyoda, K.; Hamada, I.; Lee, K.; Yanagisawa, S.; Morikawa, Y. Density Functional Theoretical Study of Pentacene/Noble Metal Interfaces with van der Waals Corrections: Vacuum Level Shifts and Electronic Structures. J. Chem. Phys. 2010, 132, 134703.

(S6) Frisch, M. J.; Trucks, G. W.; Schlegel, H. B.; Scuseria, G. E.; Robb, M. A.; Cheeseman, J. R.;

Scalmani, G.; Barone, V.; Mennucci, B.; Petersson, G. A. et al. Gaussian 09, Revision C.01, Gaussian, Inc., Wallingford CT, 2010.

(S7) Xu, B.; Tao, N. J. Measurement of Single-Molecule Resistance by Repeated Formation of Molecular Junctions. Science 2003, 301, 1221-1223.

(S8) Zhou, X-S.; Chen, Z-B.; Liu, S-H.; Jin, S.; Liu, L.; Zhang, H-M.; Xie, Z-X.; Jiang, Y-B.; Mao, B-W. Single Molecule Conductance of Dipyridines with Conjugated Ethene and Nonconjugated Ethane Bridging Group. J. Phys. Chem. C 2008, 112, 3935-3940.

(S9) Kamenetska, M.; Quek, S Y.; Whalley, A. C.; Steigerwald, M. L.; Choi, H. J.; Louie, S. G.; Nuckolls, C.; Hybertsen, M. S.; Neaton, J. B.; Venkataraman, L. Conductance and Geometry of Pyridine-Linked Single-Molecule Junctions. J. Am. Chem. Soc. 2010, 132, 6817-6821. 
(S10) He, H. X.; Shu, C.; Li, C. Z.; Tao, N. J. J. Adsorbate Effect on the Mechanical Stability of Atomically Thin metallic Wires. Electroanal. Chem. 2002, 522, 26-32.

(S11) Murai, D.; Nakazumi, T.; Fujii, S.; Komoto, Y.; Tsukagoshi, K.; Motta, C.; Kiguchi, M. Highly Stable Au Atomic Contacts Covered with Benzenedithiol under Ambient Conditions. Phys. Chem. Chem. Phys. 2014, 16, 15662-15666. 